\title{
Risk of contagion of SARS-CoV-2 among otorhinolaryngologists in Spain during the "Two waves"
}

\author{
Cristina Martin-Villares ${ }^{1}\left[\right.$. Manuel Bernal-Sprekelsen ${ }^{2}\left[\right.$ (C) Carmen Perez Molina-Ramirez ${ }^{3}$. \\ Margarita Bartolome-Benito ${ }^{4}$ on behalf of COVID ORL ESP Collaborative Group
}

Received: 23 November 2020 / Accepted: 20 December 2020 / Published online: 19 January 2021

(c) The Author(s), under exclusive licence to Springer-Verlag GmbH, DE part of Springer Nature 2021

\begin{abstract}
Introduction The aim of our study was to obtain data about the contagion rate among Otolaryngologists in Spanish ENT Departments and about the clinical outcomes in positive otolaryngologists. As a secondary objective, we aim to assess the rate of contagion in the first and the second Covid-19 wave in Spain among Otorhinolaryngologists and the regional distribution by ENT-Departments.

Methods Study design and population: This is a prospective observational study in a cohort of 975 Otolaryngologists from 87 ENT Departments conducted from March 25 to November 17 in our collaborative group, COVID ORL ESP. COVID-19 polymerase chain reaction (PCR) was the diagnostic standard. Hospitalization and/or intensive care admission and mortality was recorded as non-identified data.

Results Data collected from 975 otolaryngologist from 87 Departments resulted in 157 (16.5\%) otolaryngologists testing positive for SARS-CoV-19 by RT-PCR. Important geographic differences in contagion are reported. A total of 136 (86.6\%) otolaryngologists were tested positive during the first wave and 21 (13.3\%) during the second wave. At the last cut-off point of the study only 30/87 ENT Departments (34.5\%) remained

COVID19-free and 5 Departments reported more than $50 \%$ staff members testing positive. The majority of positive tested otolaryngologists $(126 / 157 ; 80.2 \%)$ had only mild or no symptoms, 17 developed moderate symptoms $(10.8 \%)$ and 3 had pneumonia not requiring hospitalization (2\%). Five colleagues were admitted at hospital, 4 required ICU (2.5\%) and 2 colleges died from COVID-19.

Conclusions During the first wave of the SARS-CoV-19 pandemic otolaryngologists in Spain have been overall the group suffering the highest rate of contagion, particularly during the first month. Subsequently, the Spanish Ministry of Health should include otorhinolaryngologists as a high-risk group in airborne pandemics.
\end{abstract}

Keywords SARS-CoV-2 $\cdot$ COVID-19 $\cdot$ Otolaryngology $\cdot$ Morbidity $\cdot$ Mortality

Cristina Martin-Villares, Manuel Bernal-Sprekelsen contributed equally to this work.

Collaborative group affiliations and members are listed in Acknowledgements section.

All authors contributed to the study conception and design. CMV had the responsibility of the data collection and analysis. CMV and MBS wrote the manuscript and have equally contributed as main authors. MBS made final corrections. All authors have revised the manuscript and gave approval for the published version.

Cristina Martin-Villares

crismvillares@gmail.com; mmartv@unileon.es

Extended author information available on the last page of the article

\section{Introduction}

According to data from the Ministry of Health (November 12-30, 2020), with 79,771 confirmed cases, Spanish healthcare workers (HCWs) are worldwide who were the most affected by the COVID-19 pandemic [1]. One reason may be that, at the beginning of the pandemic, the National protocol recommended PCR testing was only in symptomatic HCW [2].

The nationwide Spanish seroepidemiological study 'ENE-COVID' concluded that $10 \%$ of HCWs in our country had antibodies against SARS-CoV-2, while only $5.2 \%$ antibodies were detected in the general population [3]. In another seroprevalence study, among $578 \mathrm{HCW}$ 
from a referral hospital in Barcelona, Spain, the cumulative prevalence of SARS-CoV-2 infection was $11.2 \%$ and $40 \%$ of HCWs with past or present infection had not been previously diagnosed with COVID-19 [4].

The aim of our study was to obtain data about the contagion rate among otolaryngologists, in Spanish ENT Departments and about the clinical outcomes in positive otolaryngologists. As a secondary objective, we aim to assess the rate of contagion in the first and the second Covid-19 wave in Spain among otorhinolaryngologists and the regional distribution by ENT-Departments.

\section{Methods}

\section{Study design and population}

This is a prospective observational study in a cohort of 975 otolaryngologists conducted from March 25 to November 13 . The study population was collected from our collaborative group, COVID ORL ESP, created on March 25, among 252 otolaryngologists of 87 Spanish ENT Departments, to share the information by instant messaging about tracheostomies and other issues related to COVID-19 patients [5]. The study was designed to obtain representative data at national and regional levels. Otolaryngologists of all regions participated in the study (Fig. 1).

Participants of the collaborative group collected data from all included ENT-Departments along 8 months, from March 25 to November 13. COVID-19 polymerase chain reaction (PCR) was the diagnostic standard. Date of diagnosis, the need for hospitalization and/or intensive care admission and mortality was recorded as non-identified data. The severity of clinical outcomes of otolaryngologists tested positive was scored by a scale: (1) non-hospitalized cases, (2) hospitalized cases, (3) ICU admission, and (4) death. The two COVID-19 "waves" were defined following the official MoMo data from Spain (momo.isciii. es):

-"First wave" from March 10 to May 10 (with a 66.9\% excess deaths).

- "Second wave" from September 1 to November 16 (with $16.9 \%$ excess of deaths).

\section{Results}

After two waves of the pandemic in Spain, data collected from 975 otolaryngologist from 87 Departments resulted in $157(16.5 \%)$ otolaryngologists testing positive for SARS-CoV-19 by RT-PCR. The first otolaryngologist who

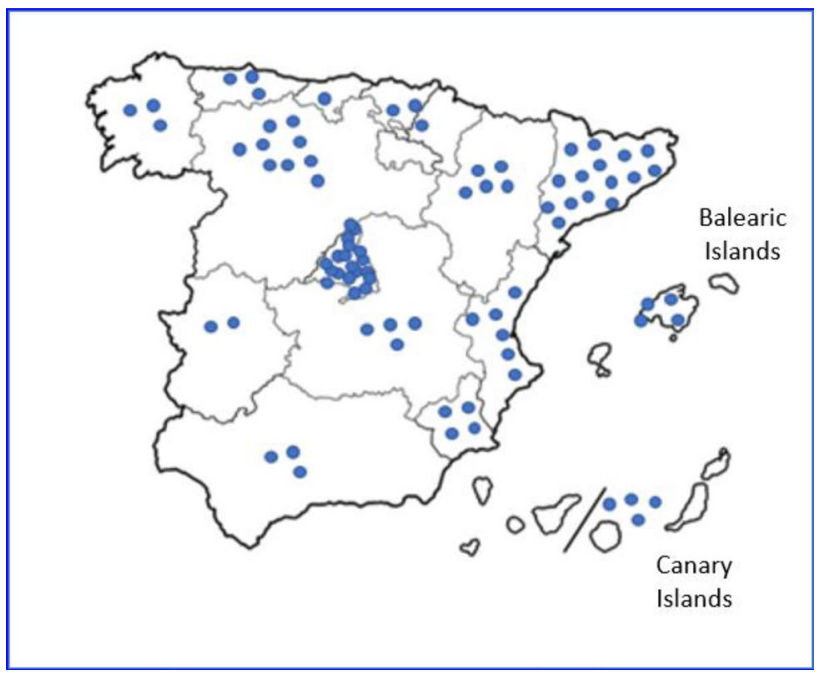

Fig. 1 Regional distribution of ENT Departments of COVID ORL ESP collaborative group

tested PCR positive was on March 9, 2020 at a hospital in Madrid. The last otolaryngologist who tested positive was on November 9 in Zaragoza Fig. 2 and Table 1.

Important geographic differences in contagion among otolaryngologists are reported in Fig. 1. Madrid, Castilla, and León and Castilla-La Mancha displayed the highest incidence of otolaryngologists who were tested positive (Table 1). The majority of positive tested otolaryngologists $(126 / 157 ; 80.2 \%)$ had only mild or no symptoms (Table 2 ). Seventeen otolaryngologists developed moderate symptoms (10.8\%) and three of them had pneumonia not requiring hospitalization (2\%). Five colleagues were admitted in hospital with moderate-severe pneumonia. Four otolaryngologists required ICU (2.5\%) for critical pneumonia and finally, one otolaryngologist died from COVID-19 in March and another colleague died in November.

A total of $136(86.6 \%)$ otolaryngologists was tested positive during the first wave and $21(13.3 \%)$ during the second wave. After the first wave, 35/87 ENT Departments of our study group (40.2\%) remained COVID19-free (Table 3). At the last cut-off point of the study, only 30/87 ENT Departments (34.5\%) remained COVID19-free and five Departments reported more than 50\% staff members testing positive. 
Table 1 Distribution of otolaryngologists testing positive by Spanish regions

\begin{tabular}{llc}
\hline Spanish regions (CA, Autonomous Community) & Otolaryngologists & \\
\cline { 2 - 3 } & 157 infected & $\begin{array}{l}975 \text { total } \\
\text { otolaryngolo- } \\
\text { gists }\end{array}$ \\
\hline CA Madrid (6 663 394 inhabitants*) & & 271 \\
CA Castilla León (2 399 548) & 58 & 102 \\
CA Castilla La Mancha (2 032 863) & 19 & 55 \\
CA Islas Canarias (2 153 389) & 18 & 64 \\
CA Cataluña (7 675 217) & 14 & 109 \\
CA Aragón (1 319 291) & 11 & 52 \\
CA País Vasco (2 207 776) & 10 & 42 \\
CA Extremadura (1 067 710) & 7 & 22 \\
CA Galicia (2 699 499) & 5 & 34 \\
CA Islas Baleares (1 149 460) & 5 & 38 \\
CA Valencia (5 003 769) & 3 & 67 \\
CA Navarra (654 214) & 3 & 24 \\
CA Asturias (1 022 800) & 3 & 25 \\
CA Cantabria (581 078) & 1 & 17 \\
CA Murcia (1 493 898) & 0 & 20 \\
CA Andalucia (8 414 240) & 0 & 33 \\
\hline
\end{tabular}

*Demographic data from Spanish National Statistics Institute (INE) in 2019 https://www.ine.es/dynt3/ inebase/es/index.htm?padre $=517 \&$ capsel $=523$

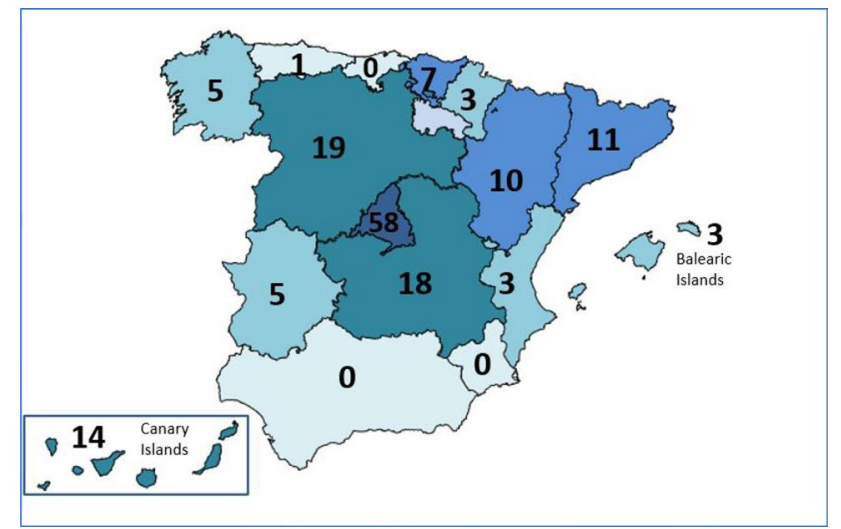

Fig. 2 Regional distribution of ENT positive tested

\section{Discussion}

The aim of our study was to obtain data about contagion among otolaryngologists and ENT Departments in Spain during the two waves of the current coronavirus pandemic, as well as potential regional differences of contagion.

Data collected over an eight-month period from 87 hospitals in Spain, showed an overall rate of contagion of $16.5 \%$, with $157 / 952$ otorhinolaryngologists testing positive for SARS-CoV-19. This rate is three times higher as compared with the general population in Spain with a seroprevalence of $5.2 \%$ of $\mathrm{IgG}$ antibodies against SARSCov2, and also above the $10 \%$ rate of antibodies among HCWs found in the same study [3]. In a large Spanish referral hospital seroprevalence of anti-bodies against SARS-CoV-2 among all HCWs reached $11.2 \%$ [4]. Data from other invasive airways physician collaborative group [6] reported a contagion rate of $10.5 \%$ among anaesthesiologists $(n=150 / 1328)$ and $12.3 \%$ among intensive care physicians $(n=31 / 251)$.

Because the study was designed to obtain representative data at both national and regional levels, we were able to observe marked regional differences in contagion, particularly between the centre of Spain (Madrid, Castilla y León, and Castilla La Mancha) and peripheral regions. Similar results about regional differences were reported in a large nationwide study about seroprevalence of SARS-CoV-2 in Spain (ENE-COVID) [3] with prevalence five times higher in Madrid and central parts of Spain than that observed in low-risk regions.

Inadequate personal protection at the beginning of the pandemic, shortage of personal protective equipment (PPE), and long-time exposure to large numbers of infected patients was established at the Shanghai International Forum as the most impact reasons for HCWs becoming infected [7]. In our study, $86 \%$ of the reported contagion happened during the first wave, coinciding with a shortage of PPEs and limited number of PCR testing performed at the beginning of the pandemic in our country. Monitoring the prevalence of 
Table 2 Clinical outcomes of otolaryngologists positive tested during pandemic (from March to November 2020)

\begin{tabular}{llll}
\hline Clinical outcomes & \multicolumn{2}{l}{$\begin{array}{l}\text { Otolaryngologist with test } \\
\text { positive for COVID-19 }\end{array}$} & \\
\cline { 2 - 3 } & \multicolumn{2}{c}{ Total $(n=157)$} & \multicolumn{1}{c}{} \\
\hline No symptoms & 11 & $7 \%$ & \\
Mild symptoms & 115 & $73.2 \%$ & $\begin{array}{c}\text { At least one symptom asso- } \\
\text { ciate with COVID-19 }\end{array}$ \\
Moderate symptoms without hospitalization & 17 & \multicolumn{2}{c}{} \\
Pneumonia without hospitalization & 3 & $10.8 \%$ & \\
Hospitalization & 5 & $2 \%$ & \\
ICU admission & 4 & $3.2 \%$ & \\
Deaths (in-Hospital) & 2 & $2.5 \%$ & 1 with neurological sequelae \\
& & $1.3 \%$ & 1 in March 2020 \\
& & & 1 in November 2020 \\
\hline
\end{tabular}

infection among different activities during pandemics will be useful for assessing the potential level of exposure and identifying high-risk diagnostic or therapeutic procedures performed by otorhinolaryngologists, thus reinforcing the critical role of PPE in our speciality to prevent contagion. Lessons learned from the SARS 2003 outbreak in Hong Kong, Singapore and Canada validated the effectiveness of standard protective personal equipment (PPE) on aerosol generating procedures in our speciality [8]. A study from one referral hospital participating in our collaborative group reported no contagion among the surgical team along with the first wave with high standards of protection in 50 COVID-19 tracheostomies [9].

Despite being otolaryngologists an obvious high-risk group, recent recommendations of the Spanish Ministry of Health about safety measurements for HCWs in aerosolgenerating procedures (AGP) left otolaryngologists out of the list of groups at risk [10], while e.g. the UK Government [11] considered any upper ENT airway procedures that involve suctioning as the potentially infectious AGPs for COVID-19 and recommended full PPE for ENT specialist while visiting any patient in the office. However, recommendations as to how physicians, and particularly otorhinolaryngologists, had to protect themselves had not been established back then. In fact, at the beginning of the pandemic PPE, let alone PAPR, were available in sufficient numbers and, as stated above, diagnostic or therapeutic procedures performed by otorhinolaryngologists have even not yet been listed as a risk. All this might contribute to explain the high rate of contagion registered during the first weeks among otorhinolaryngologists in Spain.

The results of our survey are in full contrast to a recent international study, registering Otolaryngologists-Head and Neck surgeons with COVID-19 in 19 countries [12]. Here, Spain is listed with just 11 cases for a total of 361 . The methodology of this registry remains unclear as it does not explain which inclusion period had been considered. Our contagion rate is higher than the one found in the recent national web-based survey on the SARS-CoV-2 infections among otorhinolaryngologists in Germany [13] with 54/970 (5.56\%) ORLs reported testing positive. At that point, the prevalence in the general population in Germany was lower. In any case, the German study concluded that otolaryngologists have an almost 3.7-fold risk of contracting SARSCoV-2 compared with the population baseline level, similar to our results.

The limitations of our survey are the data acquired through social media: the maximum number of participants in a WhatsApp-group is 256. Large studies also used a smartphone app to recollect prospective data on pandemic in real time [14]. Thus, we missed two additional deceased otorhinolaryngologists from hospitals not included in the study group. Also, results do not necessarily reflect the rate of nosocomial infection. However, the latter would not explain the differences in the contagion rate in comparison to other specialists handling the upper airway.

\section{Conclusions}

During the first wave of the SARS-CoV-19 pandemic otolaryngologists in Spain have been overall the group suffering the highest rate of contagion, particularly during the first month. Subsequently, the Spanish Ministry of Health should include otorhinolaryngologists as a high-risk group in airborne pandemics. 
Table 3 ENT Departments with otolaryngologist tested positive during both waves of the pandemic in Spain

\begin{tabular}{lll}
\hline $\begin{array}{l}\text { ENT Depart- } \\
\text { ments }(n=87)\end{array}$ & Otolaryngologist $+(n=157 / 952)$ \\
\cline { 2 - 3 } & $\begin{array}{l}\text { First wave }+ \text { Second } \\
\text { wave }(136+21 E N T+)\end{array}$ & $\begin{array}{l}\text { Total ENT }+ \text { at Depart- } \\
\text { ment/total ENT at Depart- } \\
\text { ment }\end{array}$ \\
& & .
\end{tabular}

\begin{tabular}{lll}
\hline D 1 & $11+2$ & $13 / 22$ \\
D 2 & $6+0$ & $6 / 14$
\end{tabular}

$\begin{array}{lll}\text { D } 3 & 6+0 & 6 / 14 \\ \text { D } 4 & 6+0 & 6 / 13 \\ \text { D } & 6+0 & 6 / 7\end{array}$

$\begin{array}{lll}\text { D } 4 & 6+0 & 5 / 78 \\ \text { D } 5 & 5+0 & 5 / 12 \\ \text { D } 6 & 3+2 & 1 / 28\end{array}$

D $7 \quad 4+0 \quad 4 / 28$

D $8+2+2 \quad 4 / 26$

D $9 \quad 3+1 \quad 4 / 22$

D $10 \quad 2+2 \quad 4 / 21$

D $114+0 \quad 4 / 16$

D $12 \quad 0+4 \quad 4 / 15$

D $134+0 \quad 4 / 15$

D $14 \quad 4+0 \quad 4 / 13$

D $15 \quad 4+0 \quad 4 / 10$

$\begin{array}{lll}\text { D } 16 & 4+0 & 4 / 7\end{array}$

$\begin{array}{lll}\text { D } 17 & 4+0 & 4 / 7\end{array}$

D $18 \quad 2+1 \quad 3 / 24$

$\begin{array}{lll}\mathrm{D} 19 & 2+1 & 3 / 21\end{array}$

$\begin{array}{lll}\text { D } 20 & 3+0 & 3 / 19\end{array}$

$\begin{array}{lll}\text { D } 21 & 3+0 & 3 / 15\end{array}$

$\begin{array}{lll}\text { D } 22 & 3+0 & 3 / 14\end{array}$

D $23 \quad 2+1 \quad 3 / 13$

D $24 \quad 3+0 \quad 3 / 13$

D $25 \quad 2+1 \quad 3 / 13$

D $26 \quad 2+1 \quad 3 / 9$

$\begin{array}{lll}\text { D } 27 & 3+0 & 3 / 8\end{array}$

$\begin{array}{lll}\text { D } 28 & 3+0 & 3 / 7\end{array}$

D $29 \quad 3+0 \quad 3 / 3$

D $30 \quad 2+0 \quad 2 / 24$

$\begin{array}{lll}\text { D } 31 & 2+0 & 2 / 19\end{array}$

D $32 \quad 2+0 \quad 2 / 16$

D $332+0 \quad 2 / 13$

D $34 \quad 2+0 \quad 2 / 12$

D $35 \quad 3+0 \quad 3 / 10$

D $36 \quad 2+0 \quad 2 / 8$

$\begin{array}{lll}\text { D } 37 & 2+0 & 2 / 7\end{array}$

D $38 \quad 1+1 \quad 2 / 5$

D $391+0 \quad 1 / 26$

$\begin{array}{lll}\text { D } 40 & 1+0 & 1 / 19\end{array}$

D $41 \quad 1+0 \quad 1 / 17$

D $42 \quad 0+1 \quad 1 / 14$

D $43 \quad 0+1 \quad 1 / 12$

D $44 \quad 1+0 \quad 1 / 10$

D $45 \quad 0+1 \quad 1 / 10$

D 46
Table 3 (continued)

\begin{tabular}{lll}
\hline ENT Depart- & Otolaryngologist $+(n=157 / 952)$ \\
\cline { 2 - 3 } ments $(n=87)$ & $\begin{array}{ll}\text { First wave }+ \text { Second } \\
\text { wave }(136+21 E N T+)\end{array}$ & $\begin{array}{l}\text { Total ENT }+ \text { at Depart- } \\
\text { ment/total ENT at Depart- } \\
\text { ment }\end{array}$ \\
& &
\end{tabular}

\begin{tabular}{lll}
\hline D 47 & $1+0$ & $1 / 8$ \\
D 48 & $1+0$ & $1 / 8$
\end{tabular}

$1+0$

D $50 \quad 1+0 \quad 1 / 6$

D $51 \quad 1+0 \quad 1 / 6$

D $52 \quad 1+0 \quad 1 / 5$

D $53 \quad 1+1 \quad 1 / 5$

D $541+1 \quad 1 / 4$

D $55 \quad 1+0 \quad 1 / 4$

D $56 \quad 0+0 \quad 0 / 23$

$\begin{array}{lll}\text { D } 57 & 0+0 & 0 / 21\end{array}$

$\begin{array}{lll}\text { D } 58 & 0+0 & 0 / 17\end{array}$

$\begin{array}{lll}\text { D } 59 & 0+0 & 0 / 17\end{array}$

$\begin{array}{lll}\text { D } 60 & 0+0 & 0 / 15\end{array}$

$\begin{array}{lll}\text { D } 61 & 0+0 & 0 / 14\end{array}$

D $62 \quad 0+0 \quad 0 / 13$

D $63 \quad 0+0 \quad 0 / 12$

D $64 \quad 0+0 \quad 0 / 11$

D $65 \quad 0+0 \quad 0 / 8$

D $66 \quad 0+0 \quad 0 / 7$

D $67 \quad 0+0 \quad 0 / 6$

D $68 \quad 0+0 \quad 0 / 6$

D $69 \quad 0+0 \quad 0 / 6$

$\begin{array}{lll}\text { D } 70 & 0+0 & 0 / 6\end{array}$

D $71 \quad 0+0 \quad 0 / 6$

D $72 \quad 0+0 \quad 0 / 6$

D $73 \quad 0+0 \quad 0 / 5$

D $74 \quad 0+0 \quad 0 / 5$

D $75 \quad 0+0 \quad 0 / 5$

D $76 \quad 0+0 \quad 0 / 5$

D $77 \quad 0+0 \quad 0 / 4$

D $78 \quad 0+0 \quad 0 / 4$

D $79 \quad 0+0 \quad 0 / 4$

D $80 \quad 0+0 \quad 0 / 4$

D $81 \quad 0+0 \quad 0 / 3$

D $82 \quad 0+0 \quad 0 / 3$

D $83 \quad 0+0 \quad 0 / 3$

D $840+0 \quad 0 / 2$

D $850+0 \quad 0 / 2$

D $86 \quad 0+0 \quad 0 / 2$

$\begin{array}{lll}\text { D } 87 & 0+0 & 0 / 2\end{array}$

$D$ ENT department, ENT otolaryngologist, ENT + otolaryngology tested positive for COVID-19 
Acknowledgements This study is dedicated to our ENT colleges and to all the HCWs who lost their health and their lives during this pandemic.The authors thank the Spanish Society of Otorhinolaryngology and Head and Neck Surgery (SEORL-CCC). CMV would like to acknowledge Professor Jesus M Culebras MD PhD, Professor of Surgery, for improved the work in innumerable ways and for his support anytime. My grateful for the expert comments offered by Miguel Aristegui, Head of Department of Gregorio Marañón Hospital, Madrid. Affiliations of Collaborators of COVID ORL ESP Collaborative Group (listed alphabetically): Albacete General Hospital: Aranzazu PerezFernandez, Sara Alcantara-Armenteros. Altahaia Xarxa Asistencial Manresa Hospital: M Dolors Deola-Trasserra, Vanessa VillarragaCova, Azor Carreras-Alcaraz, Esther Montane-Sala; Alvarez Buylla Mieres Hospital: Esther Sota-Eguizabal; Araba Vitoria Hospital: Aihnoa Tolosa, Belen De la Iglesia, Rafael Garcia-Sardon; Basurto Bilbao Hospital: Kiara Tudela-Cabello, Aitor Zabala-LopezdeMaturana; Bellvitge Barcelona Hospital: Gabriel Huguet-Llull, Marta MesallesRuíz, Ramon Jimenez-Montoya; Burgos HUBU Hospital: Ana NavazoEguïa, Blanca Galindo-Torres; Cabueñes Gijon Hospital: Marta Fernandez-Pello, Nuria Rodriguez-Prado; Caceres Hospital: Carmen Salazar, Francisco Ramos; Can Mises Ibiza Hospital: Juan Carlos Amor-Dorado; Castellon General Hospital: Marta Faubel-Serra, Rosana Almela-Cortes; CH Navarra Hospital: María Uzcanga-Lacabe, Marisela Cardier-Suárez; Ciudad Real General Hospital: Esther ÚbedaFernández, Miguel Ángel Alañon-Fernández; San Carlos Madrid Clinic Hospital: Mari Cruz Iglesias-Moreno, Jesus Gimeno-Hernández, Sara Tamboura; Salamanca Clinic Hospital: Angel Muñoz-Herrera, Marta Calvo; Valladolid Clinic Hospital: Jaime Santos-Pérez; Zaragoza Clinic Hospital: Guillermo Gil-Grasa, Maria Jose Lavilla-Martin de Valmaseda, Jose Miguel Sebastian. Ciudad de Coria Hospital: Carlos Sanchez-Herrero; Henares Coslada Hospital: Beatriz Molina-Montes, Raquel De la Fuente-Hernandez; Cruces Barakaldo Hospital: Jose Antonio Municio-Martin, Luis Pascua-Gomez; Cruz Roja Madrid Hospital: Sergio Andrino-Martin, Alejandra Ayala; CUN Madrid Hospital: Bárbara Molina-Gil, Jorge De Abajo-Larriba; 12 de Octubre Madrid Hospital: Alfredo Garcia-Fernandez, Ignacio Jimenez-Huerta; Dr Negrin Gran Canaria Hospital: Jesus Benitez-del Rosario, Alberto Sanchez-Tudela; Dr Peset: Jose Dalmau. Elche Alicante Hospital: Antonio Almodovar-Iniesta; El Escorial Madrid Hospital: Antonio MartinezRuiz-Coello; Esperit Sant Barcelona: Alberto Codina-Aroca; Ferrol Hospital: Jacobo Chao-Vietes; Fundacion Jimenez Diaz: Jose Miguel Villacampa-Auba, Alfonso Campos-Gonzalez, L Rubio-Yanguas, Lucia Baguena-Campos, Alberto Encinas-Vicente; Fuenlabrada Hospital: Estefanía Hernández-Garcia, Guillermo Plaza; Gregorio Marañon Madrid Hospital: Alejandro Lowy-Benoliel, Daniel PolettiSerafni, Juan Antonio Pasamontes-Pingarron, Miguel Aristegui-Ruiz, Monica Hernando-Cuñado, Rosalia Souviron-Encabo, Tomas Martinez-Guirado, Mario Fernandez-Fernandez, Ricardo Gonzalez-Orus; Catalunya General Hospital: Valery Nuñez-Carrasco, Adriana AgüeroMedina, Juan Carlos-Villatoro, Roser Lopez-Diu, Selvyn GonzalezMelgan; Alicante General Hospital: Manuela Sancho-Mestre, Oscar Alemán-Lopez, Irene Monjas-Cánovas; Valencia General Hospital: Enrique Zapater-Latorre, Jose Ramon Alba-Garcia, Miriam Natsuki Oishi-Konari, Beatriz Pallares-Marti, Andrea Rubio-Fernandez; Infantil Universitario Niño Jesus de Madrid: Angel Moreno-Juara, Enrique Guillén-Lozada, Saturnino Santos-Santos, Maria Jesús-Dominguez; Infanta Elena Huelva Hospital: Jesús Crovato-Rojas; Infanta Elena Valdemoro Madrid Hospital: Cristian Ruminot, Daniella Laguado, Hander Acosta, María José Hernández-Garcia; Infanta Leonor Madrid Hospital: Alejandro Zuaza-Gonzalez, Carlos Domingo-Carrasco; Infanta Sofía Madrid Hospital: Cristina Valor-García, Javier LopezMartin; Infanta Cristina Badajoz Hospital: Tomas Mogollon-Cano; Dr JMO Lanzarote Hospital: Felipe Junjgohann-Jofre, Antonio MartelLopez, Maria Dolores Martin-Sanchez; Joan XXIII Tarragona
Hospital: Carla Meler-Claramonte, Juan Carlos Flores-Martín; La Candelaria Tenerife Hospital: Juan Jose Artazkoz-del Toro; La Fe Valencia Hospital: Alfonso Garcia-Piñero, Noelia Muñoz-Fernandez; La Paz Madrid Hospital: Antonio del Palacio, Blanca Mateos-Serrano, Isabel Garcia-Lopez, Laura Rodrigañez-Riesco, Paula Aragon, Teresa Rivera; J Manuel Morales; La Princesa Madrid Hospital: Gustavo EisenbergPlaza, Inmaculada Fernandez-Robledo, Jorge Prada-Pendolero; Leon CAULE Hospital: Ignacio Alvarez-Alvarez, Maria Puente-Verez; Locus Agusti Lugo Hospital: Ana Quintana-Sanjuas, Pablo Parente-Arias; Mar Menor Murcia Hospital: Francisco García-Cordoba, Francisco Jose Garcia-Purriños; Marques de Valdecilla Santander Hospital: Carmelo Morales-Angulo; Mateu Orfila Menorca Hospital: Simara Rodriguez-Rondon, Diana Lopez-Lopez; Miguel Servet Zaragoza Hospital: Rafael Fernandez-Liesa; Militar Zaragoza Hospital: Amaya LázaroSánchez, I Alonso-Alonso; Moises Brogui Barcelona Hospital: Michalina Rusiecka, Rosa Delia Ramirez, Javier Vila; Mollet Barcelona Hospital: Angela Bellmunt-Fontanet, Victoria Montoro-Martinez; Morales Meseguer Murcia Hospital: Carlos Escobar-Sanchez; Mutua Terressa Barcelona Hospital: Gabriela Simonetti, Laura Palomino, Ignacio Clemente; Ntra Stra Sonsoles Avila Hospital: Maria Jesus Velasco; Parc Tauli Sabadell Hospital: Carlota Rovira-Ramos, Juan Jose Diaz-Argüello, Yolanda Escamilla-Carpintero; Perpetuo Socorro Badajoz Hospital: Pablo Torrico-Roman; Plato Barcelona Hospital: Carlos Calvo-Navarro, Ignacio Viza-Puiggros; El Bierzo Ponferrada Hospital: Diana Luorido, Monica Granda, Jose Miguel Tejeda; Principe Asturias Alcala Madrid Hospital: Raquel Ortiz-Rodrigo, Juan HigueraLucas, Jose Ignacio Tato, Teresa Rivera-Rodriguez; Punta de Europa Algeciras Hospital: Diego Rodriguez-Contreras, Antonio CaravacaGarcia; Quiron Salud Zaragoza Hospital: Jorge Alfaro-Garcia; Ramon y Cajal Madrid Hospital: Fatima Sanchez-Fernandez, Lourdes MontesJovellar, Mar Medina, Rafael Barbera; Reina Sofia Murcia Hospital: Alfonso MarcoGarrido, Isabel Cremades-Navalon; Rey Juan Carlos Madrid Hospital: C Garcia Bastida, Raimundo Gutierrez-Fonseca; Requena Valencia Hospital: Javier García. Rio Hortega Valladolid Hospital: Elisa Gil-Carcedo, Javier Herrero-Agustín, Jorge Freijanes-Otero, Mario Cuetos-Azcona; San Agustin Aviles Hospital: Carmen RosalFraga; San Pau i Sta Tecla Tarragona Hospital: Fabian Alzate-Amaya; San Pau Barcelona Hospital: Cesar Orus; Sant Joan de Reus: María Foglia-Fernandez, Coia Romeu-Figuerola; Sanitas La Zarzuela Madrid Hospital: Estefanía Hernández-Garcia, Laura Palomino, Marta Alcaraz; San Juan de Dios Barcelona Hospital: Cristina A Vázquez-Romero; Santiago de Compostela Hospital: Cristina Dios-Loureiro, Isabel Gonzalez-Guijarro; Segovia Hospital: Rosa Sancho-Calvo; Severo Ochoa Leganes Madrid Hospital: Jesus Jose Ramos-Fernandez, Jose Carlos Casqueiro, Mar Lasso-delaVega, Paula Martinez-Pascual, Silvia Dominguez-Ovejas; Son Espases Mallorca Hospital: Eduardo MoreraSerna, Jacoba Alba-Mesquida; Son Llatzer Mallorca Hospital: Alfonso Bonilla-Perez; Santa Barbara Soria Hospital: Javier Martinez-Subias; Sureste Arganda del Rey Madrid Hospital: Jesus M Martinez-Salazar; Talavera de la Reina Hospital: Antonio Martinez-Lapeña; Toledo Virgen de la Salud Hospital: Rocio Corrales-Millan, Rosario RuizGomez; Urduliz Vizcaya Hospital: Laura Gerarda Cianci-Jaimes; Virgen Arreixaca Murcia Hospital: Ruben Jara-Rubio; Virgen del Rocio Sevilla Hospital: Estefanía Lozano, Isabel Tirado-Zamora; Vic Hospital: Albert Idigora-Planas; Villaba General Hospital: Alvaro Sanchez-Barrueco, Ignacio Alcala-Rueda; Zamora Virgen de la Concha Hospital: Enrique Coscaron-Blanco, Soledad Suarez-Ortega; University Hospitals of Derby and Burton Nottigham (GB)* invited hospital, Hazem Nijim. Hospital General Regional 46 IMSS Jalisco (Mexico)* invited hospital Irma Yolanda Castillo-Lopez. Universidad Europea Madrid: Maria Jose Gonzalez-Gimeno. University of Leon: Ana Carvajal-Urueña, Jesus Ignacio Dominguez-Calvo, Manolo Gonzalo-Orden. 


\section{References}

1. Update No. 250. Coronavirus disease (COVID-19) (2020) Alert and Emergency Coordination Centre, Spain https://www.mscbs .gob.es/profesionales/saludPublica/ccayes/alertasActual/nCov/ documentos/Actualizacion_250_COVID-19.pdf

2. Health Ministry of Spain (2020) Procedure for action against cases of infection with the new coronavirus (SARS-CoV-2). file:///C:/ Users/asus/Downloads/Procedimiento-GENERAL-adapt2019nCoV-11-4-20\%20(2).pdf

3. Pollán M, Pérez-Gómez B, Pastor-Barriuso R, Oteo J et al (2020) Prevalence of SARS-CoV-2 in Spain (ENE-COVID): a nationwide, population-based seroepidemiological study. Lancet 396(10250):535-544. https://doi.org/10.1016/S0140 $-6736(20) 31483-5$

4. Garcia-Basteiro AL, Moncunill G, Tortajada M et al (2020) Seroprevalence of antibodies against SARS-CoV-2 among health care workers in a large Spanish reference hospital. Nat Commun 11:3500. https://doi.org/10.1038/s41467-020-17318

5. Martin-Villares C, Perez Molina-Ramirez C, Bartolome-Benito M, Bernal-Sprekelsen M, COVID ORL ESP collaborative group (2020) Outcome of 1890 tracheostomies for critical COVID19 patients: a national cohort study in Spain. Eur Arch Otorhinolaryngol. https://doi.org/10.1007/s00405-020-06220-3

6. El-Boghdadly K, Wong DJN, Owen R et al (2020) Risks to healthcare workers following tracheal intubation of patients with COVID-19: a prospective international multicentre cohort study. Anaethesia. https://doi.org/10.1111/anae.15170

7. Shanghai International Forum for Infection Control and Prevention (2020) Rational, scien-tific, and standardized protection: the core of infection prevention and control of COVID-19 in medical institutions. https://mp.weixin.qq.com/s/G5Nwdd9kW9yVDhTdwsKtg
8. Tay JK, Khoo ML, Loh WS (2020) Surgical considerations for tracheostomy during the COVID-19 pandemic: lessons learned from the severe acute respiratory syndrome outbreak. JAMA Otolaryngol Head Neck Surg. https://doi.org/10.1001/jamao to.2020.0764.10.1001/jamaoto.2020.0764

9. Avilés-Jurado FX, Prieto-Alhambra D, González-Sánchez N et al (2020) Timing, complications, and safety of tracheotomy in critically Ill patients with COVID-19. JAMA Otolaryngol Head Neck Surg. https://doi.org/10.1001/jamaoto.2020.3641

10. Health Ministry of Spain (2020) Recommendations for patient safety and professionals in interventional procedures in the transition phase of COVID-19pandemia. https://www.mscbs.gob.es/ profesionales/saludPublica/ccayes/alertasActual/nCov-China/ documentos/COVID19_Procedimientos_intervencionistas.pdf

11. GOV.UK. COVID-19: infection prevention and control. Accessed 3 May 2020. https://www.gov.uk/government/publications/wuhan -novel-coronavirus-infection-prevention-and-control-decon

12. Sowerby LJ, Stephenson K, Dickie A et al (2020) International registry of otolaryngologist-head and neck surgeons with COVID19. Int Forum Allergy Rhinol 10(11):1201-1208. https://doi. org/10.1002/alr.22677

13. Herzog M, Beule AG, Lüers JC, Guntinas-Lichius O, Sowerby LJ, Grafmans D (2020) Results of a national web-based survey on the SARS-CoV-2 infectious state of otorhinolaryngologists in Germany. Eur Arch Otorhinolaryngol 8:1-9. https://doi.org/10.1007/ s00405-020-06345-5.PMID:32897443;PMCID:PMC7477736

14. Nguyen LH, Drew DA, Graham MS et al (2020) Risk of COVID19 among front-line health-care workers and the general community: a prospective cohort study. Lancet Public Health 5(9):e475e483. https://doi.org/10.1016/S2468-2667(20)30164-X

Publisher's Note Springer Nature remains neutral with regard to jurisdictional claims in published maps and institutional affiliations.

\section{Authors and Affiliations}

\section{Cristina Martin-Villares ${ }^{1}\left(\right.$ Manuel Bernal-Sprekelsen $^{2}\left(\mathbb{D} \cdot\right.$ Carmen Perez Molina-Ramirez $^{3}$. Margarita Bartolome-Benito ${ }^{4}$ on behalf of COVID ORL ESP Collaborative Group}

1 Department of Otorhinolaryngology, Anatomy of Department of Medicine, Surgery and Anatomy, Hospital Universitario El Bierzo, University of León, Médicos sin Fronteras s/n. 24401-Ponferrda, León, Spain

2 Chair of ORL, Head of ORL-Dept., University of Barcelona, Hospital Clinic, Barcelona, Spain
3 Department of Otorhinolaryngology, Complejo Universitario Hospitalario de Segovia, University of Valladolid, Valladolid, Spain

4 Department of Otorhinolaryngology, Hospital Infantil Universitario Niño Jesús, Autonomous University of Madrid, Madrid, Spain 